\title{
Impact of left ventricular unloading using a transfemoral micro-axial pump in eCPR patients
}

\author{
Christopher Gaisendrees ${ }^{1}$, Ilija Djordjevic ${ }^{1}$, Anton Sabashnikov ${ }^{2}$, Christoph Adler ${ }^{3}$, Kaveh \\ Eghbalzadeh $^{4}$, Borko Ivanov ${ }^{3}$, Sebastian Walter ${ }^{1}$, Georg Schlachtenberger ${ }^{5}$, Julia Merkle ${ }^{3}$, \\ Ahmed Elderia $^{1}$, Stephen Gerfer ${ }^{5}$, Henning Carstens ${ }^{1}$, Antje Deppe ${ }^{2}$, Elmar W. Kuhn ${ }^{6}$, \\ and Thorsten Wahlers ${ }^{3}$ \\ ${ }^{1}$ Uniklinik Koln \\ ${ }^{2}$ University of Cologne \\ ${ }^{3}$ University Hospital Cologne \\ ${ }^{4}$ Cologne Heart Center \\ ${ }^{5}$ Klinikum der Universitat zu Koln Klinik und Poliklinik fur Herz- und Thoraxchirurgie \\ ${ }^{6}$ Heart Center, University of Cologne
}

November 2, 2020

\begin{abstract}
Objectives: Extracorporeal cardiopulmonary resuscitation (eCPR) is a rapidly growing treatment strategy due to increasing survival rates in selected patients. Additional left ventricular mechanical unloading, using a transfemoral micro-axial blood pump (Impella@), might improve patients' outcomes. In this regard, we sought to investigate patients who suffered OHCA (out- of hospital cardiac arrest) or IHCA (in-hospital cardiac arrest) with subsequent eCPR (extracorporeal cardiopulmonary

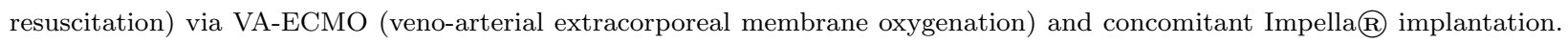
Methods: From January 2016 until December 2019, 71 patients underwent eCPR at our institution. Data prior eCPR and early outcome parameters were analyzed comparing patients who were supported with an additional transfemoral micro-axial blood pump (ECMO+Impellaß, n= 7) and patients without additional (ECMO, n=64) support during VA-ECMO therapy. Results: Baseline data did not significantly differ between groups. All-cause mortality was significantly lower in the ECMO+Impella $\mathbb{R}$ group $(83 \%$ vs. $29 \%, \mathrm{p}=0.01)$. The time of circulatory support was shorter in the ECMO+Impellaß $)$ cohort (3.16 \pm 2.09 vs. $6.5 \pm 2.79, \mathrm{p}=0.01)$. Additionally, ECMO weaning was significantly more feasible in patients with ECMO+Impella@ (71\% vs.

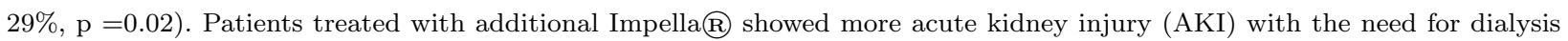
( $71 \%$ vs. $21 \%, p=0.09)$. Conclusion: From our data, concomitant Impella@ support might increase survival and successful ECMO weaning in eCPR patients. Treatment associated complications such as AKI were more common in this highly selected patient group. Further studies with larger numbers are necessary to evaluate concomitant LV-unloading's clinical relevance in eCPR patients, using an Impellaß device.
\end{abstract}

\section{Introduction}

The frequency of extracorporeal cardiopulmonary resuscitation (eCPR), either in out-of-hospital or inhospital cardiac arrest (OHCA / IHCA) scenarios, is increasing due to advances in interdisciplinary treatment of these patients (1). Moreover, mortality rates have been shown to be improving in recent years, despite still relatively low overall-survival (2). In particular, eCPR remains a specialized rescue-therapy and is mainly performed in high volume centers $(3,4)$.

In eCPR patients, left ventricular function might be massively impaired due to myocardial infarction (MI), decompensated heart insufficiency (HI), pulmonary embolism (PE), or a primary arrhythmogenic event. 
Furthermore, experimental data show that due to its retrograde flow, VA-ECMO causes left ventricular (LV) distension and an increase in left ventricular end-diastolic volume (LVEDV), left ventricular end-systolic volume (LVESV), as well as increased myocardial wall stress (5). The potential formation of an LV-thrombus is a fatal consequence of impaired and dilated LV without sufficient ejection (6).

Several strategies are being discussed to avoid or limit left ventricular dilation (7). Up to now, there are no particular indications for LV-unloading regimes and no specific guidelines (8). The decision for LVunloading is depending on clinical parameters, such as echocardiographic measures, radiological findings, and the hemodynamic situation (9). The question of routine implementation of LV unloading or selection of appropriate parameters and patients is the subject of a widespread scientific work in recent literature (9-11).

In clinical practice, short-term left ventricular assist with Impella $\mathbb{R}$ is frequently used for LV- unloading in ECMO patients (11). Besides beneficial hemodynamic effects, differences in favorable clinical outcomes might be affected by the underlying disease.

In this regard, we sought to analyze the effect of concomitant Impella $($ implantation in eCPR patients in order to analyze the impact on short-term outcomes.

\section{Patients and Methods}

From January 2016 until December 2019, 71 patients underwent eCPR at our institution. They were retrospectively analyzed and divided into two groups: ECMO $(\mathrm{n}=64)$ and

ECMO+Impella? $(n=7)$. The investigation was approved by the institutional review board of the University of Cologne and conformed to the principles outlined in the Declaration of Helsinki. Due to its retrospective study, the need for written informed consent was waived.

eCPR protocol University Hospital Cologne

The interdisciplinary eCPR team of the University Hospital Cologne is running by the Department of Cardiothoracic Surgery and the Department of Cardiology. In situations of ongoing CPR, physicians of both divisions are involved in further treatment. ECMO (Maquet, Rastatt, Germany) implantations are performed in the catheterization laboratory, intensive care unit (ICU), or emergency room. After a general clinical examination and initial echocardiographic evaluation, the left or right groin is sterilized and prepared for ECMO implantation. Additional concomitant blood gas analysis is performed with the view to assessing initial metabolic parameters. ECPR is performed in the interdisciplinary consensus of the participating disciplines. The decision to cannulate is based on participating physicians' judgment, following institutional criteria:

eCPR inclusion criteria

- No terminal illness

- Age [?] 75 years

- Observed cardiac arrest

- No-flow-time [?] 5 mins

- Low-flow-time [?] 90 mins

- refractory shockable rhythm or non-shockable in case of cardiac tamponade or pulmonary embolism

eCPR exclusion criteria

arterial $\mathrm{pH}<6.6$

non-shockable rhythm $>20$ mins after examination for cardiac tamponade or pulmonary embolism

hemoglobin $<8 \mathrm{~g} / \mathrm{dl}$

ECMO cannulas are implanted via femoral vessels in Seldinger's technique, as already published by our study group (12-15). Extracorporeal circulation is established by using a Rotaflow centrifugal pump (Maquet, 
Rastatt, Germany) with a Quadrox membrane oxygenator (Maquet, Rastatt, Germany) and the PLS2050 circuit system (Maquet, Rastatt, Germany).

After initiation of ECMO flow and stabilization of hemodynamics, the next steps of treatment were initiated.

Moreover, to avoid thromboembolic events during ECMO circulation, our anticoagulatory protocol included the intravenous infusion of unfractionated heparin, with a target activated clotting time (ACT) of 180 to 200 seconds and PTT between 60 to 80 seconds.

Laboratory data were drawn along with initial blood gas analysis or after the patient's transfer to ICU.

\section{Definitions}

Low flow time was defined as the time of advanced CPR until the commencement of ECMO circulation. Renal failure was defined as a rise in serum creatinine levels of more than $0.3 \mathrm{mg} / \mathrm{dL}(26.5 \mu \mathrm{mol} / \mathrm{L})$ over 48 hours or a rise in serum creatinine 1.5 times higher than average levels or newly developed reduction of urine at $<0.5 \mathrm{~mL} / \mathrm{kg} / \mathrm{h}$ over $6 \mathrm{~h}$.

Implementation of concomitant Impella@ support

Impella $($ ) (Abiomed, Danvers, Massachusetts, USA) implantation was realized either simultaneously with VA-ECMO in the heart catheter laboratory or after ECMO implantation on the intensive care unit, due to clinical findings as mentioned above.

- End diastolic LV-size (LVEDD) [?] $6.8 \mathrm{~cm}$ (male), $6.1 \mathrm{~cm}$ (female)

- spontaneous contrast in TTE/TEE

- Reticular lung edema in chest x-ray

Impella(r) implantation was guided by transoesophageal echocardiography. Additional Impella(r) therapy resulted in a target ACT of 180-220.

Variables of interest

The analysis was performed using a retrospectively maintained institutional patient database. The variables evaluated included: patient demographics (age, height, weight, sex), patients' status before ECMO support CPR, duration of CPR, the cause of OHCA/IHCA, CPR characteristics, and initial blood gas analysis), early outcome data ( $\mathrm{pH}$ and lactate after 12 and 24 hours, blood transfusions, in-hospital mortality, discharge, hospital, and ICU stay. As well as analyzation of end-organ damaging; acute kidney injury (AKI) requiring dialysis, stroke, bleeding, and ischemia.

\section{Endpoints of the study}

The primary endpoints of our analysis were early outcomes after initiation of eCPR and in-hospital mortality.

\section{Statistical methods}

Statistical analysis was performed using Statistical Package for Social Sciences, version 26.0 (SPSS IBM, Chicago, Illinois). All data were presented as continuous or categorical variables. Categorical data were expressed as total numbers and percentages. Continuous data were evaluated for normality using a one-sample Kolmogorov-Smirnov test and were expressed as the mean +- standard deviation (SD) in normally distributed or median (interquartile range) in cases of non-normally distributed continuous variables. Univariate analysis was performed using either Student t or Mann-Whitney U test for normally and non-normally distributed continuous variables, respectively. Pearson's $\chi^{2}$ or Fisher exact tests were used to comparing categorical data depending on the minimum expected count in each cross-tab. $\mathrm{P}$ values $<0.05$ were considered statistically significant. We performed a linear regression analysis with ANOVA for further analysis of the variable's effect. Subgroup analysis of male gender was performed.

\section{Results}

Patient's baseline characteristics before eCPR 
Baseline demographics are presented in Table 1. The ECMO group accounted for 64 patients, of which 53 were male (82.5\%) and 11 females (17.5\%). The ECMO+Impella@ group included seven male (100\%) patients. The median age was $59(55 ; 65)$ years in the ECMO group and 56(42;64) years in the ECMO+Impellaß group $(\mathrm{p}=0.32)$.

Scenarios of eCPR did differ between the groups (OHCA: 86\% ECMO+Impella $\AA$ ) vs. $39 \%$ ECMO, p=0.21; IHCA: $14 \%$ ECMO+Impella@ vs $38 \% \mathrm{ECMO}, \mathrm{p}=0.21)$. Low-flow time was 60 minutes on average in both groups.

\section{Laboratory parameters before eCPR}

Laboratory parameters before eCPR are presented in Table 2. There were no statistically significant differences between both groups.

\section{Early-Outcomes}

Early outcomes are summarized in Table 3 . Lactate and $\mathrm{pH}$ values showed no significant difference in the comparison of both groups over the first 24 hours. ECMO duration was significantly shorter in the ECMO group than in the ECMO+Impellaß) group (3.16 \pm 2.09 days ECMO vs. $6.4 \pm 2.79$ days ECMO+Impellaß,

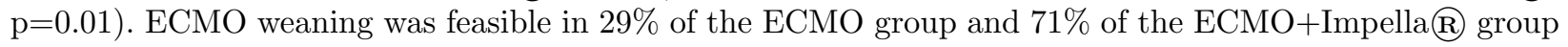
$(\mathrm{p}=0.02)$. The rate of blood transfusions differed significantly between both groups $(26(13 ; 38)$ units EC$\mathrm{MO}+\mathrm{Impella}(\mathrm{R})$ vs. $10(3,25 ; 18)$ units ECMO, $\mathrm{p}=0.01)$. The rate of transfused fresh frozen plasma units did not differ between the groups (ECMO+Impellaß $10(2 ; 15)$ and ECMO $2(0 ; 7.75), \mathrm{p}=0.09)$. The EC$\mathrm{MO}+\operatorname{Impella}(\mathbb{R}$ group received significantly more platelet units $(3(1 ; 4)$ vs ECMO group $0(0 ; 1), \mathrm{p}<0.01)$.

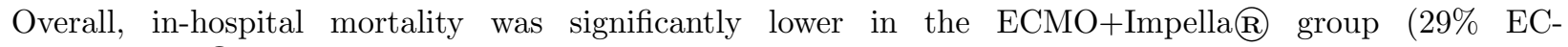
$\mathrm{MO}+\mathrm{Impella}(\mathrm{B}$ vs. $83 \% \mathrm{ECMO}, \mathrm{p}=0.01)$. The survival for both groups is presented in the Kaplan-Meier plot (Figure 1).

End organ failure

Data regarding end-organ failure are presented in Table 3. Significantly more renal failure requiring dialysis was observed in the ECMO+Impella@ group $(72 \%$ vs. $21 \%, \mathrm{p}=0.09)$. Stroke rates did not show significant differences between the groups (ECMO $21 \%$ vs. ECMO+Impella@ $29 \%, p=0.82$ ). Subsequent limb ischemia occurred in 6 cases in the ECMO group and 1 in the ECMO+Impellaß group $(\mathrm{p}=0.92)$.

\section{Linear regression and correlation analysis}

Linear regression showed a significant effect of Impella $(\mathrm{R})$ implantation on in-hospital mortality $\left(\mathrm{R}^{2}=0.15\right.$, ANOVA $\mathrm{p}=0.01,95 \% \mathrm{CI}=-0.853--0.232)$, ECMO weaning $\left(\mathrm{R}^{2}=0.08\right.$, ANOVA $\mathrm{p}=0.014,95 \% \mathrm{CI}=0.092$ $-0.805)$, and renal failure requiring dialysis $\left(\mathrm{R}^{2}=0.13\right.$, ANOVA $\left.\mathrm{p}=0.01,95 \% \mathrm{CI}=0.12-0.93\right)$. The need for red blood units was also significantly associated with Impella@ $\mathrm{Rupport}\left(\mathrm{R}^{2}=0.17,16.13295 \%\right.$ CI 7.57 24.6, $\mathrm{p}=0.00)$. Data were presented in Table 4 .

Subgroup analysis for male sex

After adjusting and comparing both groups concerning gender, the baseline parameter did not show significant differences (Table 5). There was a significantly higher rate of acute kidney injury (AKI) leading to dialysis in the ECMO+Impella $($ ) group, compared with the ECMO group (71\% vs. 25\%, p=0.03). Also, the ECMO+Impella $(\mathbb{B})$ group received significantly more red blood units $(26$ vs. $10, \mathrm{p}=0.01)$ and platelets $(0$

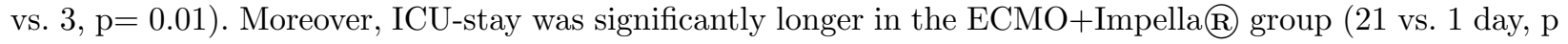
$=0.03)$. Overall mortality was significantly lower in the ECMO+Impella $®$ group $(29 \%$ vs. $85 \%, p=0.01)$.

\section{Discussion}

Our retrospective analysis, comparing simultaneous mechanical support with ECMO+Impella@ versus single

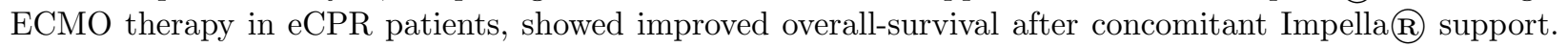
From the data presented, Impella $\mathbb{B}$ assist was associated with a higher weaning rate of ECMO support. 
The association of lower mortality and a higher rate of successful ECMO weaning was consistent through all investigated subgroups, including a comparison of only male patients. On the other hand, complications, including AKI with subsequent hemodialysis, were more frequently seen in the ECMO+Impellaß group.

ECMO-therapy establishes an artificial cardiopulmonary bypass in severely impaired hemodynamic situations, such as ongoing CPR, and provides adequate oxygenation for vital organs (16). Besides, ECMO therapy is associated with various complications of inadequate treatment, especially the increased afterload, which might negatively impact the outcome (17).

In this retrospective analysis, we observed a significant reduction in in-hospital mortality in the ECMO+Impella (B) cohort, alongside a significantly higher rate of successful ECMO weaning in the EC$\mathrm{MO}+$ Impella $(\mathrm{B}$ cohort. These findings are supported by a recent large multicenter cohort study by Schraege and colleagues, who analyzed data from 686 cardiogenic shock patients treated with VA-ECMO with or without left ventricular unloading by concomitant Impella@ implantation (11). In their observational analysis, simultaneous Impella@ implantation was associated with significantly lower mortality in cardiogenic shock and eCPR patients and more complications, such as bleeding, ischemia, and renal replacement therapy. Another broad analysis by Pappalardo et al. showed similar results, describing increased AKI rates with hemodialysis (18).

The increased survival rates observed in our cohort might be explained by the potential benefits of LVunloading in eCPR patients. Previously, early LV-unloading has been reported to be beneficial in cardiogenic shock patients treated with ECMO via the reduction in preload $(17,19)$.

Other mechanical support devices might also improve mortality and outcome. An intraaortic balloon pump might also be beneficial concomitant to VA-ECMO therapy in eCPR patients due to its counter pulsatile fashion (20).

On the other hand, other authors and centers advocate a conservative strategy regarding additional Impella $\mathbb{R}$. implantation in VA-ECMO therapy $(9,10)$. Today, there is no universally used unloading strategy; thus, LVventing in VA-ECMO patients remains to be the target of ongoing scientific research. Randomized trials are necessary to confirm the reduction in mortality going along with LV unloading and determining a certainty, strategy-wise, for eCPR patients.

\section{Safety of concomitant Impella @-placement}

In our retrospective analysis, complications such as AKI and the need for blood-transfusions occurred more

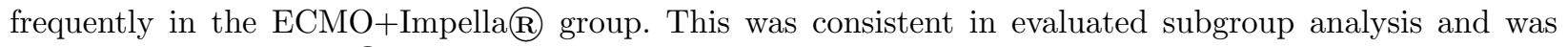
associated with Impellaß-therapy in linear regression analysis.

A higher rate of active bleeding or limb ischemia could not be observed.

This confers to larger evaluations, such as Schraege et al., who observed a higher rate of complications such

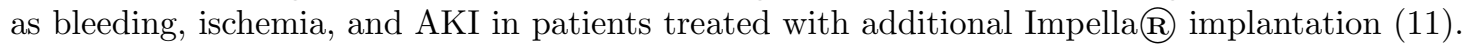

In regard to an increase of shear stress, hemolysis is a known complication associated with Impella@ and is associated with an increased need for blood-transfusions (21). As well as limb ischemia due to large vascular access requirements of Impella $®$ support.

In this analysis, we found a significantly higher proportion of AKI with the need for hemodialysis in the EC-

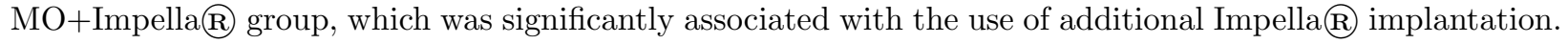
ECMO therapy is a known risk factor for renal failure (22-24). Moreover, it is thought to lead to a reduction in renal oxygen delivery and inflammatory damage. In particular, during pathological conditions requiring ECMO, biological defense mechanisms maintaining central perfusion by reducing perfusion of peripheral organs (such as the kidney) have been identified. However, the role of additional Impella@ implantation on acute kidney failure remains unclear and clinical data is scarce (25-27).

In summary, complications like the higher need for renal replacement therapy, bleeding, and limb ischemia 
might be explained by more increased survival. Thus, patients are more exposed to the risk of suffering complications.

\section{Study limitations}

This study was limited by its small sample size (Impella@ group $n=7$ ). Patients with Impella@ might have been clinically and hemodynamically more stable, also underlined by significantly longer survival and lower mortality. Hence, clinical parameters like the need for blood transfusions and ECMO weaning are challenging to interpret in the ECMO+Impellaß group. Also, hemodynamic parameters from bedside echocardiography are not captured in this analysis, and thus we cannot analyze differences in LV-distension parameters objectively between groups.

\section{Conclusion}

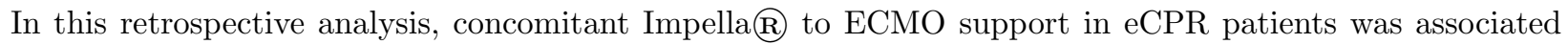
with significantly lower in-hospital mortality. Also, weaning of VA-ECMO was more often successful in the ECMO+Impella@ group. ECMO+Impella(B) group showed significantly higher rates of blood transfusions and acute kidney failure requiring dialysis.

The realization of a simultaneous mechanical circulatory assist device might be a beneficial therapy option in eCPR patients. Further studies are needed to evaluate the clinical relevance and impact of Impella $\mathbb{R}$ devices in eCPR patients.

\section{Tables and figures:}

Tables

Table 1. Baseline demographic data.

Table 2. Laboratory parameters.

Table 3. Outcomes and end-organ damage.

Table 4. Linear regression.

Table 5. Subgroup analysis of the male gender.

Figures

Figure 1. Kaplan-Meier survival.

Table 1. Baseline demographic data.

\begin{tabular}{llll}
\hline Parameter & ECMO $(\mathbf{n}=\mathbf{6 4})$ & $\begin{array}{l}\text { ECMO+Impella } \mathbb{R} \text { (n } \\
\mathbf{7}\end{array}$ & p-value \\
\hline Male & $53(82.5 \%)$ & $7(100 \%)$ & 0.10 \\
Age & $59(55 ; 65)$ & $56(42 ; 64)$ & 0.32 \\
OHCA & $39(62 \%)$ & $6(86 \%)$ & 0.21 \\
IHCA & $24(38 \%)$ & $1(14 \%)$ & 0.21 \\
Low-flow time (min) & $60(45 ; 75)$ & $60(55 ; 75)$ & 0.95 \\
Lactate prior & $12.65(9.65 ; 16)$ & $18.84(12.68 ; 18.5)$ & 0.33 \\
$\begin{array}{l}\text { implantation } \\
\text { (mmol/l) }\end{array}$ & & & \\
pH prior & & 6.88 & 0.24 \\
implantation & 6.98 & & \\
\hline
\end{tabular}

Table 2. Laboratory parameters. 


\begin{tabular}{llll}
\hline Parameter & ECMO $(\mathbf{n = 6 4})$ & $\begin{array}{l}\text { ECMO+Impella } \mathbb{R} \\
(\mathbf{n = 7})\end{array}$ & p-value \\
\hline $\begin{array}{l}\text { Urea admission } \\
\text { (mg(dL) }\end{array}$ & $42(32 ; 63)$ & $30(25.75 ; 48)$ & 0.26 \\
$\begin{array}{l}\text { Creatinine } \\
\text { admission }(\mathbf{m g} / \mathbf{d L})\end{array}$ & $1.32(1.0 ; 1.86)$ & $1.44(1.0 ; 3.75)$ & 0.79 \\
$\begin{array}{l}\text { Bilirubin admission } \\
\text { AST admission }\end{array}$ & $0.4(0.25 ; 0.5)$ & $0.6(0.55 ; 0.82)$ & 0.02 \\
ALT admission & $399(117 ; 668)$ & $456(259 ; 857)$ & 0.46 \\
CK admission & $167(72 ; 384)$ & $159(86.25 ; 304)$ & 0.79 \\
CK ater 24h & $305(220 ; 2865)$ & $3406(1716 ; 5132)$ & 0.08 \\
CK-MB \% & $16.5(1195 ; 5769)$ & $6012(1974 ; 8778)$ & 0.35 \\
$\begin{array}{l}\text { admission } \\
\text { CK-MB admission }\end{array}$ & $195(87 ; 415)$ & $14(10 ; 180)$ & 0.49 \\
$\begin{array}{l}\text { Platelets admission } \\
\text { pH 12h after }\end{array}$ & $163(110 ; 244)$ & $376(27 ; 659)$ & 0.52 \\
$\begin{array}{l}\text { implantation } \\
\text { pH 24h after }\end{array}$ & $7.38(7.33 ; 7.43)$ & $176(106 ; 200)$ & 0.91 \\
$\begin{array}{l}\text { implantation } \\
\begin{array}{l}\text { Lactate 12h after } \\
\text { (mmol/l) }\end{array}\end{array}$ & $7.42(7.36 ; 7.46)$ & $7.33(7.29 ; 7.47)$ & 0.50 \\
$\begin{array}{l}\text { Lactate 24h after } \\
\text { (mmol/l) }\end{array}$ & $6.67(3.79 ; 13)$ & $7.44(7.38 ; 7.50)$ & 0.42 \\
\hline
\end{tabular}

Table 3. Outcomes and end-organ damage.

\begin{tabular}{|c|c|c|c|}
\hline Parameter & $\operatorname{ECMO}(\mathrm{n}=64)$ & $\begin{array}{l}\text { ECMO+Impella }(\mathbb{R})(\mathrm{n} \\
=7)\end{array}$ & p-value \\
\hline $\begin{array}{l}\text { ECMO duration } \\
\text { (days) }\end{array}$ & $3.1 \pm 2.09$ & $6.4 \pm 2.79$ & 0.01 \\
\hline $\begin{array}{l}\text { Cases of ECMO } \\
\text { weaning }\end{array}$ & $19(29 \%)$ & $5(71 \%)$ & 0.02 \\
\hline $\begin{array}{l}\text { AKI requiring } \\
\text { dialysis }\end{array}$ & $13(21 \%)$ & $5(71 \%)$ & 0.09 \\
\hline Limb ischemia & $6(9.5 \%)$ & $1(14 \%)$ & 0.92 \\
\hline stroke & $13(21 \%)$ & $2(29 \%)$ & 0.82 \\
\hline Red blood (units) & $10(3.25 ; 18)$ & $26(13 ; 38)$ & 0.01 \\
\hline FFP(units) & $2(0.7 ; 7.5)$ & $10(2 ; 15)$ & 0.09 \\
\hline Platelets (units) & $0(0 ; 1)$ & $3(1 ; 4)$ & 0.00 \\
\hline $\begin{array}{l}\text { In-hospital } \\
\text { mortality }\end{array}$ & $53(83 \%)$ & $2(29 \%)$ & 0.01 \\
\hline
\end{tabular}

Table 4. Linear regression.

\begin{tabular}{lllll}
\hline Parameter & Regression & $\mathbf{9 5 \%}$ CI & $\mathbf{R}^{\mathbf{2}}$ & p-value \\
\hline In-hospital mortality & -0.542 & -0.853 to -0.232 & 0.15 & 0.01 \\
ECMO weaning & 0.449 & 0.092 to 0.805 & 0.84 & 0.01 \\
Red blood units & 16.132 & 7.57 to 24.69 & 0.17 & 0.00
\end{tabular}




\begin{tabular}{lllll}
\hline Parameter & Regression & $\mathbf{9 5 \%}$ CI & $\mathbf{R}^{\mathbf{2}}$ & p-value \\
\hline Platelets & 1.94 & 0.862 to 3.03 & 0.15 & 0.01 \\
AKI requiring dialysis & 0.531 & 0.12 to 0.93 & 0.13 & 0.01 \\
\hline
\end{tabular}

Table 5. Subgroup analysis of the male gender.

\begin{tabular}{|c|c|c|c|}
\hline Parameter & ECMO, male $(n=53)$ & $\begin{array}{l}\text { ECMO+Impella }(\mathbb{R}, \\
\text { male }(\mathrm{n}=7)\end{array}$ & p-value \\
\hline Age & $60(46 ; 66)$ & $56(42 ; 64)$ & 0.30 \\
\hline OHCA & $35(66 \%)$ & $6(86 \%)$ & 0.29 \\
\hline IHCA & $18(34 \%)$ & $1(14 \%)$ & 0.29 \\
\hline Low-flow time (min) & $60(46.25 ; 77.5)$ & $60(55 ; 75)$ & 0.90 \\
\hline $\begin{array}{l}\text { Lactate prior } \\
\text { implantation } \\
(\mathrm{mmol} / \mathrm{l})\end{array}$ & $12.64(10 ; 16)$ & $18.84(12.68 ; 18.5)$ & 0.34 \\
\hline $\begin{array}{l}\text { pH prior } \\
\text { implantation }\end{array}$ & 6.91 & 6.88 & 0.39 \\
\hline $\begin{array}{l}\text { ECMO duration } \\
\text { (days) }\end{array}$ & $3.64 \pm 1.64$ & $6.4 \pm 2.79$ & 0.02 \\
\hline $\begin{array}{l}\text { cases of ECMO } \\
\text { weaning }\end{array}$ & $13(25 \%)$ & $5(71 \%)$ & 0.12 \\
\hline $\begin{array}{l}\text { AKI requiring } \\
\text { dialysis }\end{array}$ & $13(25 \%)$ & $5(71 \%)$ & 0.03 \\
\hline Limb ischemia & $4(8 \%)$ & $1(14 \%)$ & 0.77 \\
\hline Stroke & $12(23 \%)$ & $2(29 \%)$ & 0.93 \\
\hline Red blood units & $10(2.5 ; 18)$ & $26(13 ; 38)$ & 0.01 \\
\hline FFP & $2(0 ; 8.5)$ & $10(2 ; 15)$ & 0.10 \\
\hline Platelets & $0(0 ; 1)$ & $3(1 ; 4)$ & 0.01 \\
\hline $\begin{array}{l}\text { In-hospital } \\
\text { mortality }\end{array}$ & $45(85 \%)$ & $2(29 \%)$ & 0.01 \\
\hline
\end{tabular}

Figure 1. Kaplan-Meier survival (log-rank $\mathrm{p}=0.01)$. 


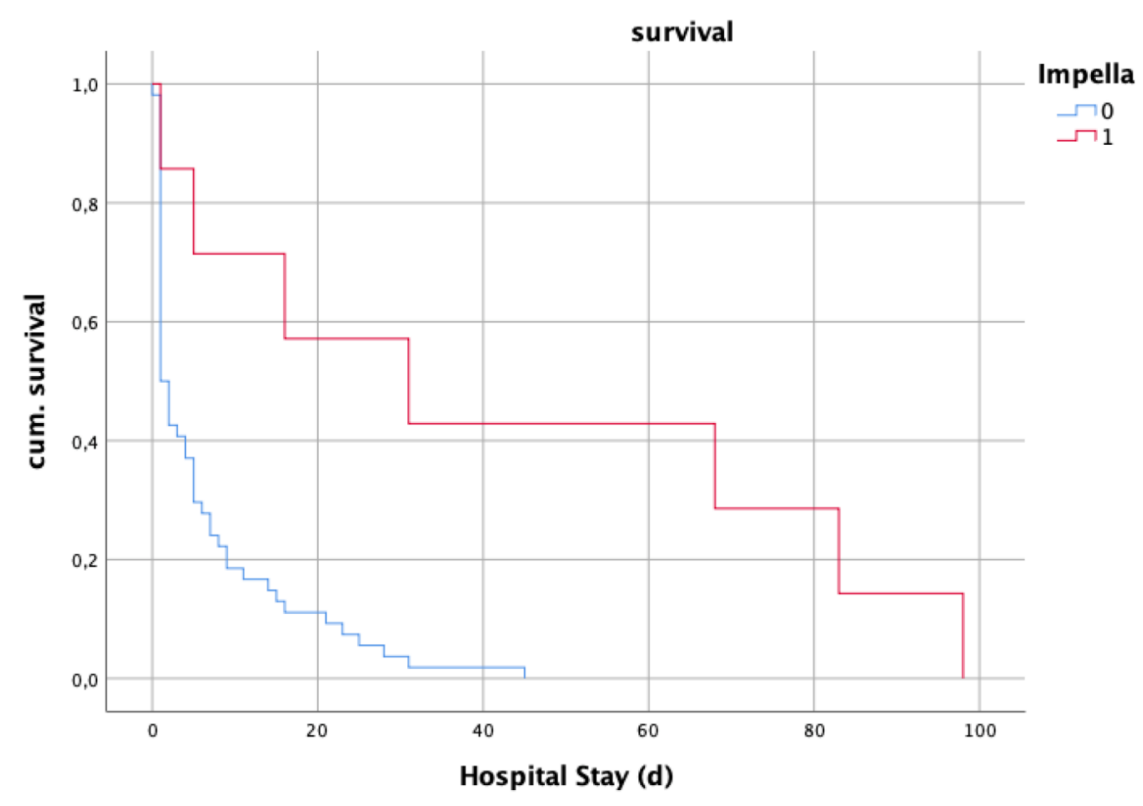

\section{References}

1. Hryniewicz K, Hart M, Raile D, Wang Y, Mooney M, Mudy K, et al. Multidisciplinary shock team is associated with improved outcomes in patients undergoing ECPR. The International Journal of Artificial Organs.0(0):0391398820962807.

2. Kim H, Cho YH. Role of extracorporeal cardiopulmonary resuscitation in adults. Acute and critical care. 2020;35(1):1-9.

3. Wang GN, Chen XF, Qiao L, Mei Y, Lv JR, Huang XH, et al. Comparison of extracorporeal and conventional cardiopulmonary resuscitation: A meta-analysis of 2260 patients with cardiac arrest. World J Emerg Med. 2017;8(1):5-11.

4. Lin JW, Wang MJ, Yu HY, Wang CH, Chang WT, Jerng JS, et al. Comparing the survival between extracorporeal rescue and conventional resuscitation in adult in-hospital cardiac arrests: propensity analysis of three-year data. Resuscitation. 2010;81(7):796-803.

5. Schrage B, Burkhoff D, Rübsamen N, Becher PM, Schwarzl M, Bernhardt A, et al. Unloading of the Left Ventricle During Venoarterial Extracorporeal Membrane Oxygenation Therapy in Cardiogenic Shock. JACC Heart Fail. 2018;6(12):1035-43.

6. Schiller P, Vikholm P, Hellgren L. Experimental Venoarterial Extracorporeal Membrane Oxygenation Induces Left Ventricular Dysfunction. Asaio j. 2016;62(5):518-24.

7. Russo JJ, Aleksova N, Pitcher I, Couture E, Parlow S, Faraz M, et al. Left Ventricular Unloading During Extracorporeal Membrane Oxygenation in Patients With Cardiogenic Shock. Journal of the American College of Cardiology. 2019;73(6):654-62.

8. Organization E-ELS. Guidelines for Adult Cardiac Failure. 2013.

9. Truby LK, Takeda K, Mauro C, Yuzefpolskaya M, Garan AR, Kirtane AJ, et al. Incidence and Implications of Left Ventricular Distention During Venoarterial Extracorporeal Membrane Oxygenation Support. Asaio j. 2017;63(3):257-65.

10. Camboni D, Schmid C. To vent or not on veno-arterial extracorporeal membrane oxygenation, does it improve myocardial recovery and outcome? J Thorac Dis. 2017;9(12):4915-8. 
11. Schrage B, Becher PM, Bernhardt A, Bezerra H, Blankenberg S, Brunner S, et al. Left Ventricular Unloading is Associated with Lower Mortality in Cardiogenic Shock Patients Treated with Veno-Arterial Extracorporeal Membrane Oxygenation: Results From An International, Multicenter Cohort Study. Circulation. 2020.

12. Djordjevic I, Sabashnikov A, Deppe AC, Kuhn E, Eghbalzadeh K, Merkle J, et al. Risk factors associated with 30-day mortality for out-of-center ECMO support: experience from the newly launched ECMO retrieval service. Journal of Artificial Organs. 2019;22(2):110-7.

13. Sabashnikov A, Djordjevic I, Deppe AC, Kuhn EW, Merkle J, Weber C, et al. Managing Traps and Pitfalls During Initial Steps of an ECMO Retrieval Program Using a Miniaturized Portable System: What Have We Learned From the First Two Years? Artif Organs. 2018;42(5):484-92.

14. Gaisendrees C, Djordjevic I, Sabashnikov A, Adler C, Eghbalzadeh K, Ivanov B, et al. Gender-related differences in treatment and outcome of eCPR-patients. Artif Organs. 2020.

15. Gaisendrees C, Suhr L, Ivanov B, Wörmann J, Djordjevic I, Eghbalzadeh K, et al. Using hydrophilic introducer sheath for peripheral vaECMO cannulation in highly calcified vessels: the bailout solution. J Artif Organs. 2020.

16. Thiele H, Ohman EM, de Waha-Thiele S, Zeymer U, Desch S. Management of cardiogenic shock complicating myocardial infarction: an update 2019. Eur Heart J. 2019;40(32):2671-83.

17. Uriel N, Sayer G, Annamalai S, Kapur NK, Burkhoff D. Mechanical Unloading in Heart Failure. J Am Coll Cardiol. 2018;72(5):569-80.

18. Pappalardo F, Schulte C, Pieri M, Schrage B, Contri R, Soeffker G, et al. Concomitant implantation of Impella ( $($ ) ) on top of veno-arterial extracorporeal membrane oxygenation may improve survival of patients with cardiogenic shock. Eur J Heart Fail. 2017;19(3):404-12.

19. Tongers J, Sieweke JT, Kühn C, Napp LC, Flierl U, Röntgen P, et al. Early Escalation of Mechanical Circulatory Support Stabilizes and Potentially Rescues Patients in Refractory Cardiogenic Shock. Circ Heart Fail. 2020;13(3):e005853.

20. Thiele H, Zeymer U, Neumann F-J, Ferenc M, Olbrich H-G, Hausleiter J, et al. Intraaortic Balloon Support for Myocardial Infarction with Cardiogenic Shock. New England Journal of Medicine. 2012;367(14):128796.

21. Badiye AP, Hernandez GA, Novoa I, Chaparro SV. Incidence of Hemolysis in Patients with Cardiogenic Shock Treated with Impella Percutaneous Left Ventricular Assist Device. ASAIO J. 2016;62(1):11-4.

22. Askenazi DJ, Selewski DT, Paden ML, Cooper DS, Bridges BC, Zappitelli M, et al. Renal replacement therapy in critically ill patients receiving extracorporeal membrane oxygenation. Clin J Am Soc Nephrol. 2012;7(8):1328-36.

23. Lin CY, Chen YC, Tsai FC, Tian YC, Jenq CC, Fang JT, et al. RIFLE classification is predictive of short-term prognosis in critically ill patients with acute renal failure supported by extracorporeal membrane oxygenation. Nephrol Dial Transplant. 2006;21(10):2867-73.

24. Yan X, Jia S, Meng X, Dong P, Jia M, Wan J, et al. Acute kidney injury in adult postcardiotomy patients with extracorporeal membrane oxygenation: evaluation of the RIFLE classification and the Acute Kidney Injury Network criteria. Eur J Cardiothorac Surg. 2010;37(2):334-8.

25. Ooi YS, Gray WA. Taking the kidney to heart. Catheter Cardiovasc Interv. 2020;95(6):1122-3.

26. Fahad F, Saad Shaukat MH, Yager N. Incidence and Outcomes of Acute Kidney Injury Requiring Renal Replacement Therapy in Patients on Percutaneous Mechanical Circulatory Support with Impella-CP for Cardiogenic Shock. Cureus. 2020;12(1):e6591. 
27. Markus B, Patsalis N, Chatzis G, Luesebrink U, Ahrens H, Schieffer B, et al. Impact of microaxillar mechanical left ventricular support on renal resistive index in patients with cardiogenic shock after myocardial infarction: a pilot trial to predict renal organ dysfunction in cardiogenic shock. Eur Heart J Acute Cardiovasc Care. 2020;9(2):158-63. 